\title{
Interactive comment on "Seasonal variation of atmospheric particle number concentrations, new particle formation and atmospheric oxidation capacity at the high Arctic site Villum Research Station, Station Nord” by Q. T. Nguyen et al.
}

\section{Anonymous Referee \#1}

Received and published: 19 May 2016

\section{Overview:}

This study presents aerosol size distribution data collected at VRS Nord. The main focus is on new particle formation. The subject is well within the scope of ACP, and especially new particle formation remain a challenge to resolve in this region.

Although the topic is relevant indeed,in its current form the MS suffers from several shortcomings.

General comments: 
The analysis performed is in many aspects superficial, and does not leave much support to the conclusions presented. Thus, the MS need substantial improvements prior final publication. My main concerns are listed below, followed by more detailed comments.

I suggest providing a more detailed description of the whole data set. The authors has a large number of events to derive statistics from, but still only treats 3 specific events in detail. It is ok ta have case studies, but I do miss the general picture regarding timing of events, role of ozone and trajectory analysis. What is the role of mass and surface during onset of events?

The analysis of the role of ozone is superficial and is apparently based on three case studies. In the MS, it seems that the role of ozone is discussed in an unjustified way. More detailed analysis is required to create support statements regarding the role of 03.

The interpretation of the trajectory analysis regarding the three cases A-C is conceptually wrong. Suggestions for improvements of analysis is given under specific comments below.

Currently, two of the main conclusions provided by the authors lacks adequate support from the analysis performed. In its current form, it is not recommended to proceed with publication of the MS.

However, as information regarding new particle formation is scarce in the Arctic, as also acknowledged by the authors, I do not want to reject the MS completely. However, major revisions is needed prior publication, taking into account the comments given below.

Recommendation: Major revision

Printer-friendly version

Specific comments:

Discussion paper

Page 2, line 3: Not true. Accumulation mode is apparently present, but in low concen- 
trations.

Page 4, line 15: Unsure if "while" is proper word here.

Page 4, line 20: "Details" $\rightarrow$ "detail"

Page 5, Instrumentation: I assume that correction for diffusion losses and CPC efficiency is taken into account during the inversion, but suggest that it is explicitly stated as the paper is dealing with new particle formation.

Page 5, Instrumentation: Please provide specification of the inlet used.

Page 6,line 9-10: Sentence awkward, suggest rewording.

Page 7, line 19: What data is actually used in this study, only 2012 or the period 20102012.cf page 6, line 13.

Page 7, Figure 4: I suggest adding some range to the data in the figure, i.e. percentile ranges in some suitable range, i.e. 25th-75th percentile to give a better view of variability.

Figure 4: Caption: Should say "table 1", not "table 2" if text is referring to lognormalparameters. Please also specify how the fitting was done

Page 8, line 15: Why does the figure "especially" describe the accumulation mode? Suggest rewording

Table 2: Is there a special reason for selecting a density of $1.4 \mathrm{~g} / \mathrm{cm} 3$

Page 8, line 26-29: Suggest rewording. As it reads now, it seems that the nucleation mode concentration is attributed to high accumulation mode concentration.

Page 10, line 27: Suggest replace "high" with "strong" or similar.

Page 10, line 29: At what altitude above ground is the anemometer placed. How does topography around the site of observation affect the quality of observations? Why weren't trajectories used to classify the transport? 
Page 10, line 31: What do the authors mean by a "broader region"?

Page 11, section 3.2.1: I suggest that the authors provide a table or figure showing ACPD timing of onset of nucleation, a simple frequency diagram would suffice. This will show if there is any diurnal preferences regarding onset of NPF.

Page 12, line 4-5: It is unclear what the authors mean here. Figure 9 does not show any details about ozone, why it is difficult to follow the authors reasoning. I strongly suggest that the authors provide a more detailed evaluation of the role of O3 in NPF or tracer thereof.

Table 3: The numbers do not add up: I assume that data is divided in three main classes: events, non-events and unclassified. Together these should add up to $100 \%$. However, reading the table, March has $83 \%$ total, April $92 \%$ total, May $112 \%$ total. Thus, it needs to be clarified to what the percentage is referring to if not to total amount of observations!?

Page 12, line 15-16: It seems that the station is within a cloud/fog. Is the inlet whole air or does it have a well defined cut off?

Page 12, line 24-29: Suggest deleting this paragraph as it has nothing to do with NPF.

Page 13, line 13-15: What does the author mean by "allowing the ozone to recover"? The reasoning seem to assume that the growth control ozone and not vice versa... To me, it seems like a change in air-mass.

Page 13, line 16-20. This is a quite shaky statement. The variation in ozone and relation formation/growth is not unambiguous. Further, the statement is based on three cases. With less than the authors back the discussion with more statistics, to me the role of ozone seems speculative to say the least. Thus, I strongly suggest that the relation to ozone is studied more in detail. In its current form however, the limited amount of data presented doesn't support anything.

Printer-friendly version

Page 14, line 2-4: The author discuss the role of mass (or rather surface) in regulating 
amount of small particles via coagulation. Of higher concern would be the role of the condensation sink (CS) of both nucleating and condensing species.

Page 14, first section: Is the correlation derived for the integral number or for number concentration over some representative size interval? If the latter is not true, I suggest an integration over nuclei mode size range, e.g. 10-30nm, prior attempting correlation tests.

Page 14, line 28-31: I do not follow completely. As I read the text, low ozone hinders NO-NO2 transition. So far so good. However, then the author state: "Local NOx emissions thus seemed to relate to...". What do the authors mean?? What is the connection?

Page 15, line 8: “. . . at the expense of NOx concentration. .." I was under the impression that the role of $\mathrm{NOx}$ in ozone formation is the photolysis of $\mathrm{NO} 2$ to $\mathrm{NO}+\mathrm{O}$. Thus, ozone formation does not reduce total levels of NOx.

Page 15, line 26: On how big fraction did this occur? How is the boundary layer defined?

Whole discussion using trajectories: The approach using the trajectories is conceptually wrong. All four examples is calculated for trajectory arrival around 01:00 at the end of the event. How does that at all relate to the onset/evolution of the event. In order to be able to discuss anything about the role of the air-mass to some aerosol property, arrival time of trajectory and observation of the parameter must coincide in time. The problem in reasoning is exemplified by e.g. Page 15, lines 28 -onwards where the authors discuss mixing along the trajectories. It has to be made clear, that the mixing taking place during 19-june in a trajectory arriving 21-june, 01:00 absolutely has nothing to do with the air arriving to the site 19-june. At least, the author has not shown that such a connection exist.

In order to do the analysis properly, I would recommend the authors to look at the 
whole time series during the event, calculate one trajectory every hour for the duration of the event. Only then aerosol properties during the event will be linkable to air-mass transport.

As currently used, the conclusions from the trajectory analysis down to page 16, line 26 , are completely redundant and should be removed or revised accordingly.

Page 18, conclusions: The conclusion that ozone is closely related to NPF is not supported by the analysis presented. Three presented cases, and a typically poor correlation between ozone and particle integral number, is clearly not enough. The same goes for the conclusions from the trajectory analysis regarding subsidence. The approach by which the authors derive the conclusion is wrong. Thus, the only verifiable outcome of the analysis is seasonality of NPF events.

Interactive comment on Atmos. Chem. Phys. Discuss., doi:10.5194/acp-2016-205, 2016. 